\title{
Impact de la Variabilité Climatique sur la Niche Ecologique de Diospyros mespiliformis Hochst. ex A.De. dans la Région Soudanienne au Bénin (Afrique de l'Ouest)
}

\author{
Karimou Soufiyanou, \\ Toko Imorou Ismailla, \\ Laboratoire de Cartographie (LaCarto), \\ Université d'Abomey-Calavi, Cotonou-Houéyiho, Bénin \\ Arouna Ousséni, \\ Laboratoire de Cartographie (LaCarto), Université d'Abomey-Calavi, \\ Cotonou-Houéyiho, Bénin. Ecole des Sciences et Techniques du Bâtiment et \\ de la Route (ESTBR), Université Nationale des Sciences, Technologies, \\ Ingénierie et Mathématiques, Bénin
}

Doi:10.19044/esj.2019.v15n36p1 ～URL:http://dx.doi.org/10.19044/esj.2019.v15n36p1

\section{Résumé}

Les espèces à usages multiples sont de plus en plus menacées par des pressions anthropiques. Ces espèces peuvent également être affectées par les variabilités climatiques qui sont de plus en plus récurrentes. La présente étude vise à analyser l'impact de la variabilité climatique sur la niche écologique de Diospyros mespiliformis dans la région soudanienne au Bénin. A cet effet, 11044 données d'occurrences de Diospyros mespiliformis ont été combinées aux variables environnementales de deux scénarii climatiques dont l'un optimiste (RCP 4.5) et l'autre pessimiste (RCP 8.5) suivant l'approche d'entropie maximale (Maxent). Dans les conditions climatiques actuelles et futures (RCP 4.5 et 8.5 ), plus de $80 \%$ de la superficie de la région soudanienne et plus de $67 \%$ de celle du réseau des aires protégées présentent des probabilités brutes d'occurrence de Diospyros mespiliformis supérieur ou égale 0,5. Cela montre que dans l'ensemble, les conditions écologiques et climatiques actuelles et futures de la région soudanienne sont propices à la culture et à la conservation de Diospyros mespiliformis à l'horizon 2055. Il paraît alors impérieux de mener des investigations sur le potentiel de régénération de cette espèce dans les aires protégées pour l'identification des sites prioritaires à sa culture et conservation dans le future. La prise en compte de ces résultats dans la politique forestière devrait contribuer efficacement à la conservation durable de Diospyros mespiliformis. 
Mots clés: D. mespiliformis, Habitats favorables, MaxEnt, Région Soudanienne, Bénin

\title{
Climate Variability Impact on Diospyros mespiliformis Hochst. ex A.De. Ecological Niche in the Sudanian Region of Benin (West Africa)
}

\author{
Karimou Soufiyanou, \\ Toko Imorou Ismaïla, \\ Laboratoire de Cartographie (LaCarto), \\ Université d'Abomey-Calavi, Cotonou-Houéyiho, Bénin
}

Arouna Ousséni,

Laboratoire de Cartographie (LaCarto), Université d'Abomey-Calavi, Cotonou-Houéyiho, Bénin. Ecole des Sciences et Techniques du Bâtiment et de la Route (ESTBR), Université Nationale des Sciences, Technologies,

Ingénierie et Mathématiques, Bénin

\begin{abstract}
Multi-use species are increasingly threatened by anthropogenic pressures. These species can also be affected by climatic variability, which is increasingly recurrent. The purpose of this study is to analyze the impact of climate variability on Diospyros mespiliformis ecological niche in the sudanian region of Benin. To this end, 11044 data on occurrences of Diospyros mespiliformis were combined with the environmental variables of two climate scenarios, one optimistic (RCP 4.5) and the other pessimistic (RCP 8.5) following the maximum entropy approach (Maxent). Under current and future climatic conditions (RCP 4.5 and 8.5), more than $80 \%$ of the area of the sudanian region and more than $67 \%$ of the area of the protected area network have gross probabilities of occurrence of Diospyros mespiliformis greater than or equal to 0.5. This shows that, overall, the current and future ecological and climatic conditions in the Sudanian region are favourable for the cultivation and conservation of Diospyros mespiliformis at 2055. It therefore seems imperative to investigate the regeneration potential of this species in protected areas in order to identify priority sites for its cultivation and conservation in the future. Taking these results into account in forest policy should effectively contribute to the sustainable conservation of Diospyros mespiliformis.
\end{abstract}


Keywords: D. mespiliformis, Habitats modelling, MaxEnt, Sudanian region, Benin

\section{Introduction}

Diospyros mespiliformis (Ebenaceae) est un arbre à feuilles caduques confiné aux régions tropicales et subtropicales, y compris l'Afrique et l'Asie (Abdullah et al., 2014 ; Arbonier, 2002 ; Vivien et Faure, 1996 ). Le feuillage est dense et vert foncé avec des feuilles elliptiques et des fruits souvent consommés par des animaux de pâturage (Belemtougri et al., 2006) mais également par l'homme (Agbani et al., 2017 ; Arbonier, 2002). L'espèce se rencontre depuis les bosquets sahélo-soudaniens jusqu'aux forêts guinéennes, dans les galeries forestières, bords de rivières, termitières et collines rocheuses généralement sur des sols lourds et bien drainés (Arbonnier, 2002). Son bois est dur et résistant aux attaques de termites (Jegede et al., 2015 ; Arbonier, 2002). Il sert en menuiserie pour les charpentes, la confection des pilons, des mortiers, des manches d'outils et d'armes, etc. C'est une espèce dont les extraits aqueux des feuilles luttent efficacement contre le paludisme (Yolidjé et al., 2019). Des recherches antérieures ont révélé que la germination de cette espèce est naturellement lente et peu abondante surtout en période froide ; mais devient rapide quand il fait chaud (Ali et al., 2017 ; Jegede et al., 2015 ; Vivien et Faure, 1996).

Les populations de Diospyros mespiliformis sont actuellement en nette régression à cause des prélèvements et des difficultés de sa régénération (Nchoutpouen, 2003). Selon certains auteurs (Abdullah et al., 2014), cette espèce est en voie de disparition en Arabie Saoudite. Dans la région soudanienne en Afrique de l'Ouest, Zizka et al. (2015) ; Gning et al. (2013); Traoré et al. (2011) et Vodouhê et al. (2009) ont montré que cette espèce est vulnérable et fait partie des espèces qui subissent le plus, les actions anthropiques compte tenu de leurs valeurs socio-économiques. Au Bénin, Diospyros mespiliformis figure parmi les trente-etune espèces ligneuses d'importance utilisées en médecine traditionnelle et est sur la liste des huit plantes ligneuses alimentaires prioritaires à préserver (Codjia et al., 2003).

Au-delà du fait que la perte d'habitats de la flore serait actuellement due aux activités anthropiques, les changements climatiques pourraient affecter la distribution et entraîner l'extinction au cours du siècle prochain de nombreuses espèces végétales (Giam et al., 2010). Ils constituent aujourd'hui, l'un des principales menaces qui pèse sur la biodiversité (GIEC, 2013).

Or, la modélisation de la distribution des espèces représente un outil important de prédiction en écologie de la conservation (Padalia et al., 2014 ; Franklin, 2009 ; Elith et al., 2006 ; Phillips et al., 2006 et Guisan et Zimmermann, 2000). Elle permet de répondre aux problématiques majeures que sont la compréhension, la description et la prédiction de l'aire potentielle 
d'une espèce, et l'identification des facteurs qui déterminent sa distribution (Kumar et Stohlgren, 2009). La modélisation représente de ce fait, un outil pertinent pour la gestion et la conservation de l'environnement et de la biodiversité (Guisan et Zimmermann, 2000). L'objectif de la présente recherche est d'évaluer l'impact de la variabilité climatique sur la niche écologique de Diospyros mespiliformis sous l'hypothèse selon laquelle, une variation des conditions environnementales et climatiques dans l'espace et dans le temps aurait des répercussions sur l'étendue des habitats de l'espèce.

\section{Matériel et Méthodes}

\section{Milieu d'étude}

La région soudanienne au Bénin est située au nord du pays. Elle couvre entièrement les départements de l'Atacora, de l'Alibori et l'extrême nord des départements de la Donga et du Borgou. Elle est comprise entre 9 $49^{\prime} 31^{\prime \prime}$ et $12^{\circ} 24^{\prime} 48^{\prime \prime}$ de latitude nord et entre $0^{\circ} 46^{\prime} 09^{\prime}$ ' et $3^{\circ} 49^{\prime} 28^{\prime \prime}$ de longitude est. Sur le plan phytogéographique, la région soudanienne fait partie de l'une des trois principales subdivisions chorologique du Bénin (Adomou et al., 2006). Elle comprend trois districts phytogéographiques à savoir le district du Borgou-nord, de Mékrou-Pendjari et de la Chaîne de l'Atacora (figure 1). Elle couvre une superficie d'environ 57009,07 $\mathrm{km}^{2}$ et représente environ 49,46\% de la superficie du territoire national. Le climat est de type soudanien et est caractérisé par une succession dans l'année, de deux saisons dont une saison sèche et une saison des pluies. Les types de sols rencontrés résultent essentiellement du processus de ferrallitisation et de ferruginisation (Le Barbe et al., 1993 ; Viennot,1978; et Faure, 1977). La végétation naturelle est constituée d'ilots de forêt dense sèche, de forêt galerie, de forêt claire et de formations savanicoles (savanes arborée, arbustive et herbeuse). 


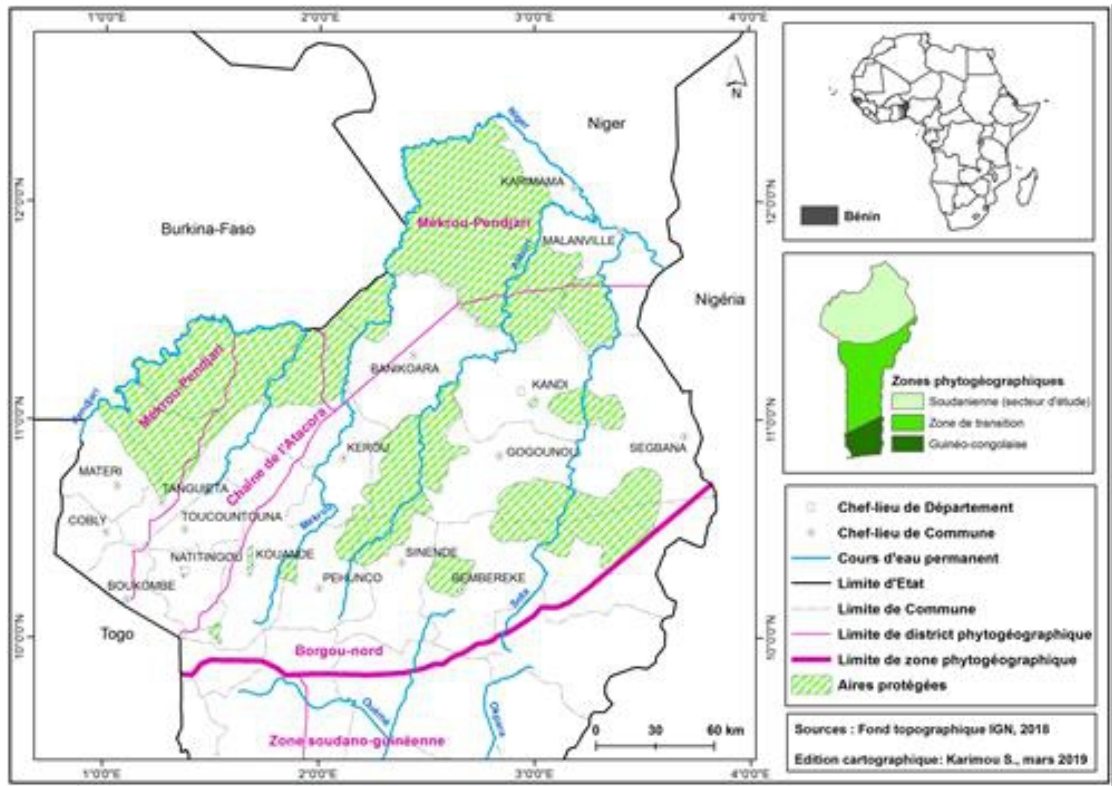

Figure 1 : Situations géographique et phytogéographique du milieu d'étude

\section{Données d'occurrence de Diospyros mespiliformis}

Les coordonnées géographiques (longitude et latitude) en degré décimal de Diospyros mespiliformis ont été collectées dans les trois phytodistricts de la région soudanienne au Bénin entre 2017 et 2019 au moyen d'un GPS. A ces données, ce sont ajoutées celles de l'Afrique de l'Ouest disponibles sur le site (http://www.gbif.org/occurrence/download) de GBIF. Au total, 11044 données d'occurrences de Diospyros mespiliformis ont été collectées (dont 3896 pour les travaux de terrain et 7148 provenant de GBIF). La figure 2 présente la spatialisation des données d'occurrence utilisées.

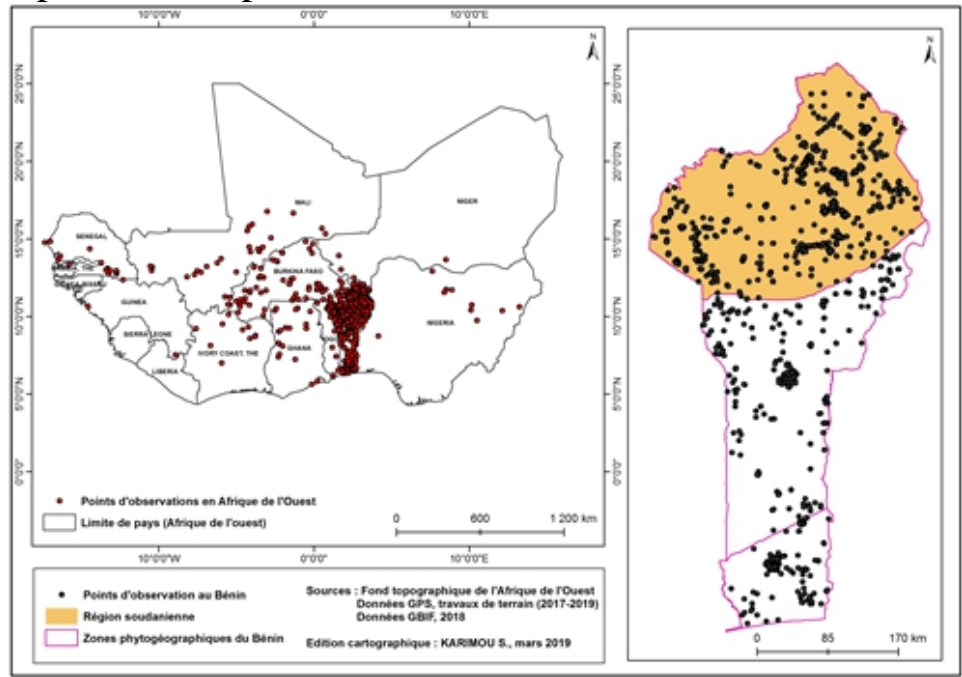

Figure 2 : Occurrences de Diospyros mespiliformis en Afrique de l'Ouest et au Bénin 


\section{Variables environnementales utilisées}

Les données sur les conditions climatiques actuelles ont été dérivées des données climatiques (1950-2000) de la base de données Worldclim version 1.4. Pour les projections climatiques futures, le modèle ensemble de circulation régionale «AFRICLIM 3.0 : highresolution ensemble climate projections for Africa sur https://webfiles.york.ac.uk/KITE/AfriClim a été utilisée. Ce modèle est plus raffiné comparativement aux modèles de circulation globale (Platt et al., 2015). Pour ce modèle, les projections à l'horizon 2055 ont été retenues sous deux scénarii : RCP 4.5 et RCP 8.5. Les couches climatiques utilisées sont celles de résolution arc 30 seconde (soit une grille de résolution d'approximativement $1 \mathrm{~km} \times 1 \mathrm{~km}$ ). Aux données bioclimatiques, il y a été ajouté les données des images Shuttle Radar Topography Mission (SRTM) (https://earthexplorer.usgs.gov) et les données relatives aux formations pédologiques (http://webarchive.iiasa.ac.at/Research/LUC/External-Worldsoil-database) à la même résolution. Le jeu de données constitué a été soumis à une analyse de corrélation afin d'éliminer les variables fortement corrélées comme l'a suggéré certains auteurs (Elith et al., 2010). Cette analyse a été faite avec l'outil ENMTools 1.3.

\section{Modélisation et évaluation du modèle}

Les données d'occurrence de terrain et de GBIF ont été combinées pour la modélisation dans le programme MaxEnt. Cette base de données constituée a été divisée en deux catégories. La première catégorie comporte $25 \%$ des données d'occurrence et est utilisée pour tester le modèle de prédiction. La seconde catégorie comportant $75 \%$ des données d'occurrence a permis de calibrer le modèle en cinq répétitions par validation croisée. Le test de Jackknife a été réalisé pour déterminer l'importance des variables environnementales individuelles utilisées. La statistique de Area Under the Curve (AUC) (Phillips et al., 2006) a été utilisée pour apprécier la performance du modèle de même que True Skill Statistique (TSS) (Allouche et al., 2006). Le modèle est dit performant si la valeur de AUC est supérieure à 0,90 ; il est passable lorsque $0,75 \leq \mathrm{AUC} \leq 0,90$ et il est qualifié de mauvais si AUC $<0,75$.

TSS est la capacité du modèle à détecter avec précision les vraies présences (sensitivité) et les vraies absences (spécificité). Un modèle avec TSS $\leq 0$ indique une prédiction aléatoire, tandis qu'un modèle avec un TSS proche de 1 (TSS > 0,5) a un bon pouvoir prédictif (Allouche et al., 2006).

\section{Cartographie des résultats de la modélisation}

Les résultats de la modélisation ont été transférés dans le logiciel ArcGIS 10.4 pour la cartographie de l'étendue des habitats de l'espèce sous 
l'effet des conditions climatiques actuelle et future. La distribution de probabilité brute obtenue par le modèle a été considérée comme mesure de la probabilité d'occurrence de l'espèce. Une catégorisation à trois niveaux de cette probabilité a été faite pour la discrimination des habitats de l'espèce à travers l'outil « raster calculator » du même logiciel. Ainsi, dans le cadre de la présente recherche, un habitat est considéré comme peu favorable lorsque la probabilité d'occurrence de l'espèce est inférieure à 0,5 . Si cette probabilité est comprise entre 0,5 et 0,7 l'habitat est moyennement favorable. Les valeurs de probabilité supérieure ou égale à 0,7 sont considérées comme habitats très favorables.

\section{Résultats}

\section{Contribution des variables à la prédiction de la niche écologique}

Les variables présélectionnées ont différemment contribuées à la prédiction des habitats dans MaxEnt. Le tableau I présente la contribution des variables à la prédiction des habitats de Diospyros mespiliformis

Tableau I : Contribution des variables à la prédiction des habitats de l'espèce

\begin{tabular}{|c|c|c|c|}
\hline $\begin{array}{c}\text { Variabl } \\
\text { es }\end{array}$ & Signification & $\begin{array}{c}\text { Contributio } \\
\text { n (\%) }\end{array}$ & $\begin{array}{c}\text { Importance de } \\
\text { permutation }(\%)\end{array}$ \\
\hline sol & Types de sols & 59,4 & 15,3 \\
\hline pet & $\begin{array}{l}\text { Evapotranspiration } \\
\text { potentielle }\end{array}$ & 24,4 & 51,6 \\
\hline bio1 & $\begin{array}{c}\text { Température moyenne } \\
\text { annuelle }\end{array}$ & 9,5 & 23,2 \\
\hline bio14 & $\begin{array}{l}\text { Précipitation du mois } \\
\text { le plus sec }\end{array}$ & 4,2 & 4,7 \\
\hline alt & Altitude & 2,5 & 5,2 \\
\hline
\end{tabular}

Source : Résultats de la modélisation

De l'examen du tableau I, on note une variation en proportion (\%) de la contribution des variables telles que types de sols (sol), évapotranspiration potentielle (pet), température moyenne annuelle (bio 1), précipitation du mois le plus sec (bio 14) et altitude (alt) à la prédiction du modèle. Les variables telles que types de sols et évapotranspiration potentielle cumulent une contribution à hauteur de $83,8 \%$ et sont par conséquent les variables qui ont le plus contribuées à la prédiction des habitats de l'espèce du point de vue de leur ordre d'intégration dans le modèle de prédiction.

Les proportions observées au niveau de la dernière colonne du tableau I sont relatives à la réduction du pouvoir prédictif du modèle lorsque les valeurs d'une variable donnée sont aléatoirement permutées entre les points du background et ceux de présence. Une valeur élevée indique une grande importance de la variable concernée.

Ainsi, en termes d'importance de permutation, la variable évapotranspiration potentielle provoque la réduction de près de $52 \%$ du 
pouvoir prédictif du modèle et celle de la température moyenne annuelle le réduit à 23,2\%. On en déduit que la permutation de ces deux variables réduit à près de 74,8 \% la prédiction du modèle de prédiction. Par conséquent, ces deux variables ont été les plus déterminantes dans la prédiction de la dynamique spatio-temporelle des habitats de Diospyros mespiliformis. Par contre, les variables telles que l'altitude et les précipitations du mois le plus sec ont faiblement influées la discrimination de ces habitats.

\section{Pouvoir prédictif du modèle et test de Jackknife}

La valeur moyenne de TSS obtenue est de 0,748 avec un écart type de 0,027. Celle de l'AUC est de 0,90 avec un écart type de 0,010. Ces valeurs indiquent la bonne performance du modèle à prédire la dynamique spatiotemporelle des habitats de Diospyros mespiliformis dans le milieu d'étude. La figure 3 présente le test de Jackknife sur l'importance des variables utilisées en termes de gain d'information.

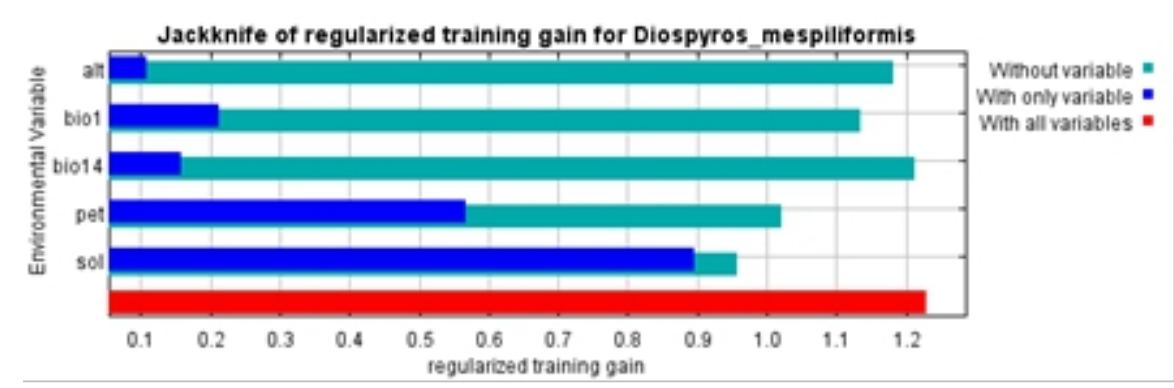

Figure 3 : Test de Jackknife sur les variables environnementales utilisées

Source : Résultats de la modélisation

De l'examen de la figure 3, il ressort que la variable qui augmente le gain d'information expliquant la distribution de Diospyros mespiliformis lorsqu'elle est isolement utilisée est le sol. Elle est suivie de l'evapotranspiration potentielle (pet) puis des valeurs de la température moyenne annuelle (bio 1). Ces trois variables sont respectivement dans l'ordre de citation, celles qui détiennent le plus d'informations non contenues dans les autres variables.

Dynamique des aires de distribution actuelle et future de Diospyros mespiliformis

La dynamique des aires de distribution actuelle et future de Diospyros mespiliformis avarié dans l'espace et dans le temps aussi bien de façon globale qu'au niveau des aires protégées.

\section{Aires de distribution actuelle et future dans la région soudanienne}

Le tableau II présente la variation de la superficie des habitats actuels et futurs de Diospyros mespiliformis et la figure 4 indique la spatialisation de ces habitats dans la région soudanienne du Bénin. 
Tableau II : Aires de distribution actuelle et future dans la région soudanienne

\begin{tabular}{ccccccc}
\hline $\begin{array}{c}D . \\
\begin{array}{c}\text { mespilifor } \\
\text { mis }\end{array}\end{array}$ & Habitats plus favorables & \multicolumn{2}{c}{$\begin{array}{c}\text { Habitats moyennement } \\
\text { favorables }\end{array}$} & \multicolumn{2}{c}{$\begin{array}{c}\text { Habitats peu } \\
\text { favorables }\end{array}$} \\
\hline & $\begin{array}{c}\text { Superficie } \\
\left(\mathrm{Km}^{2}\right)\end{array}$ & $\begin{array}{c}\text { Proportion } \\
(\%)\end{array}$ & $\begin{array}{c}\text { Superficie } \\
\left(\mathrm{Km}^{2}\right)\end{array}$ & $\begin{array}{c}\text { Proportion } \\
(\%)\end{array}$ & $\begin{array}{c}\text { Superficie } \\
\left(\mathrm{Km}^{2}\right)\end{array}$ & $\begin{array}{c}\text { Proporti } \\
\text { on }(\%)\end{array}$ \\
\cline { 2 - 7 } Présent & 36787 & 63,36 & 12197 & 21,01 & 9077 & 15,63 \\
RCP 4.5 & 35413 & 60,99 & 13471 & 23,20 & 9177 & 15,81 \\
RCP 8.5 & 35046 & 60,36 & 13514 & 23,28 & 9501 & 16,36 \\
\hline
\end{tabular}

Source : Résultats de la modélisation

De l'observation du tableau II, il ressort que la proportion de la superficie des habitats de Diospyros mespiliformis varie d'un scénario à un autre. Dans les conditions climatiques actuelles, les habitats plus favorables à la distribution de Diospyros mespilformis représentent 63,36 \% de la superficie totale du milieu d'étude. Ces habitats sont localisés au centre et s'étendent du nord-est au sud-est et une partie du sud-ouest de la région soudanienne (figure 4). Ceux moyennement favorables représentent environ $21,01 \%$ de la superficie du milieu d'étude et sont localisés du nord, à l'ouest et à l'extrême sud. Les habitats peu favorables occupent une proportion de $15,63 \%$ et se localisent à l'ouest, à l'extrême nord et une partie du sud-ouest.

Dans le cadre de l'atténuation des émissions de gaz à effet de serre (RCP 4.5), les habitats plus favorables occuperont une proportion d'environ $61 \%$ de la superficie du milieu d'étude et seront pratiquement localisés au niveau des habitats qui leurs sont actuellement plus favorables à l'exception du sud-ouest qui connaîtra une régression au profit des habitats moyennement favorables (figure 4). La proportion de la superficie des habitats peu favorables passera de 15,63 à 15,81\% à l'horizon 2055 (tableau 2).

Dans le cadre d'une augmentation des émissions de gaz à effet de serre (RCP 8.5), la proportion des habitats actuellement plus favorables passera de $63,36 \%$ à $60,36 \%$ à l'horizon 2055 (tableau 2). Cette dynamique est perceptible surtout au sud-ouest du milieu d'étude (figure 4) au profit des habitats moyennement favorables. Ce scénario indique une augmentation de la proportion des superficies des habitats moyennement et peu favorables à l'horizon 2055. 


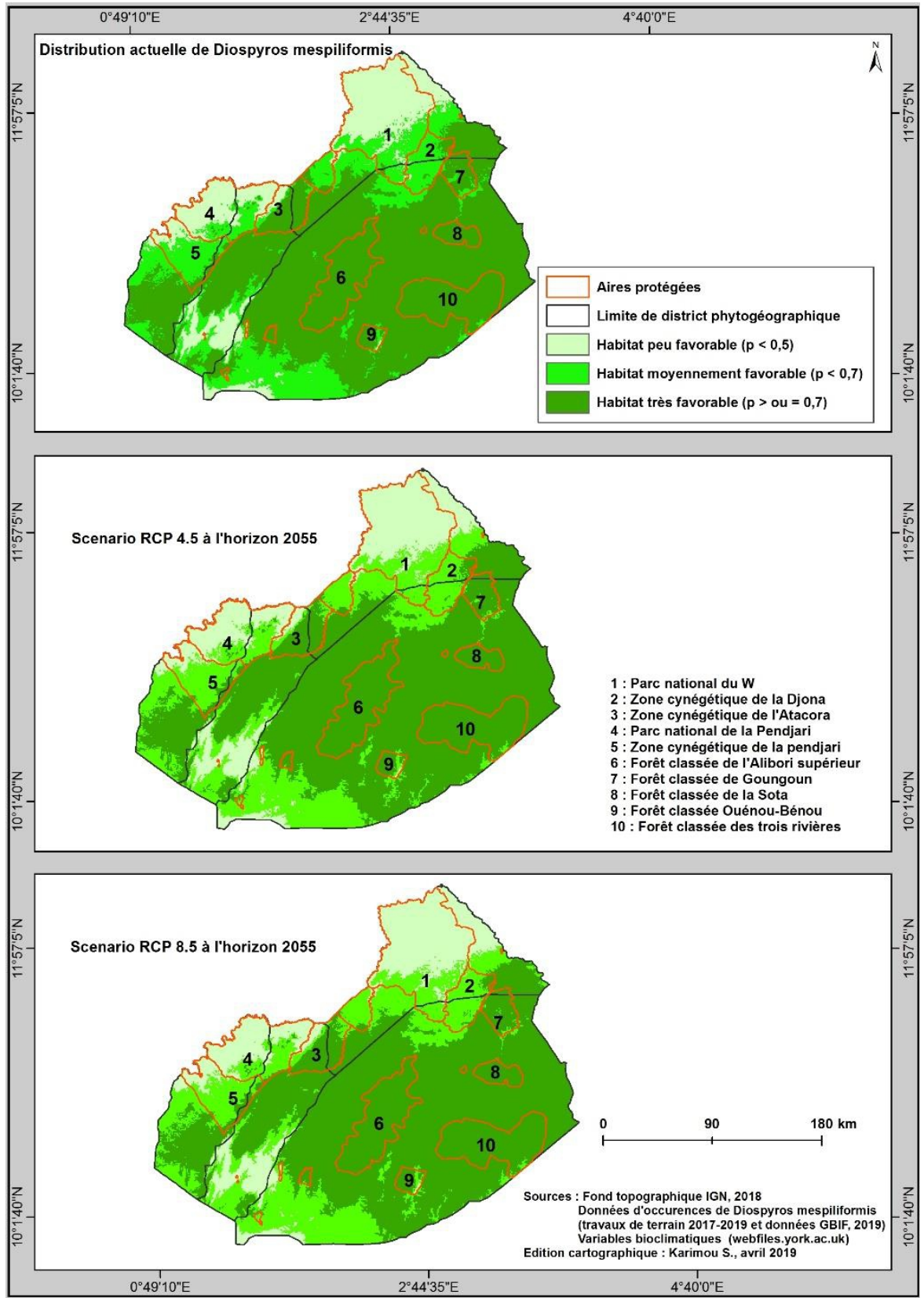

Figure 4 : Spatialisation des habitats de Diospyros mespiliformis dans la région soudanienne

Source : Résultats de la modélisation

\section{Aires de distribution actuelle et future dans les aires protégées}

Le tableau III présente la variation de la superficie des habitats actuels et futurs de Diospyros mespiliformis tandis que la figure 5 indique la spatialisation de ces habitats au niveau des aires protégées de la région soudanienne. 
Tableau III: Aires de distribution actuelle et future dans les aires protégées

\begin{tabular}{ccccccc}
\hline $\begin{array}{c}\text { D. } \\
\begin{array}{c}\text { mespilifor } \\
\text { mis }\end{array}\end{array}$ & \multicolumn{2}{c}{$\begin{array}{c}\text { Habitata plus } \\
\text { favorables }\end{array}$} & \multicolumn{2}{c}{$\begin{array}{c}\text { Habitats moyennement } \\
\text { favorables }\end{array}$} & \multicolumn{2}{c}{$\begin{array}{c}\text { Habitats peu } \\
\text { favorables }\end{array}$} \\
\hline & $\begin{array}{c}\text { Superficie } \\
\left(\mathrm{Km}^{2}\right)\end{array}$ & $\begin{array}{c}\text { Proportio } \\
\mathrm{n}(\%)\end{array}$ & $\begin{array}{c}\text { Superficie } \\
\left(\mathrm{Km}^{2}\right)\end{array}$ & $\begin{array}{c}\text { Proportion } \\
(\%)\end{array}$ & $\begin{array}{c}\text { Superficie } \\
\left(\mathrm{Km}^{2}\right)\end{array}$ & $\begin{array}{c}\text { Proportio } \\
\mathrm{n}(\%)\end{array}$ \\
\cline { 2 - 7 } Présent & 8659 & 42,89 & 5066 & 25,10 & 6462 & 32,01 \\
RCP 4.5 & 8607 & 42,64 & 5325 & 26,38 & 6255 & 30,99 \\
RCP 8.5 & 8535 & 42,28 & 5146 & 25,49 & 6506 & 32,23 \\
\hline
\end{tabular}

Source : Résultats de la modélisation

De l'examen du tableau III, il ressort que dans les conditions climatiques actuelles, la proportion des superficies plus favorables à Diospyros mespiliformis au niveau des aires protégées est 42,89\%. Ces habitats sont localisés dans les aires protégées du phytodistrict de Borgou-nord et au nord du phytodistrict de la chaîne de l'Atacora (figure 5). Les habitats moyennement favorables représentent environ $25 \%$ de la superficie des aires protégées et se rencontrent au sud du phytodistrict de Mékrou-Pendjari et sur une portion de l'extrême nord du phytodistrict du Borgou-nord. Par contre, les habitats peu favorables occupent une proportion de 32,01\% et couvrent principalement le nord du phytodistrict de Mékrou-Pendjari.

Le scénario RCP 4.5 indique que la proportion des habitats actuellement plus favorables connaîtra une légère diminution et passera de $42,89 \%$ à $42,64 \%$; de même que les habitats peu favorables. Par contre, les habitats moyennement favorables connaîtront une augmentation de la proportion de leur superficie qui passera de $25,10 \%$ à $26,38 \%$.

Le scénario RCP 8.5 indique une diminution plus prononcée $(42,28 \%)$ de la proportion de la superficie des habitats actuellement plus favorables que le RCP 4.5. Par contre, au niveau des habitats moyennement et peu favorables, on note une augmentation respective de la proportion de la superficie de ces habitats à l'horizon 2055.

Les tendances de la localisation de la dynamique des habitats des deux scénarii au niveau des aires protégées du milieu d'étude sont pratiquement similaires (figure 5). 

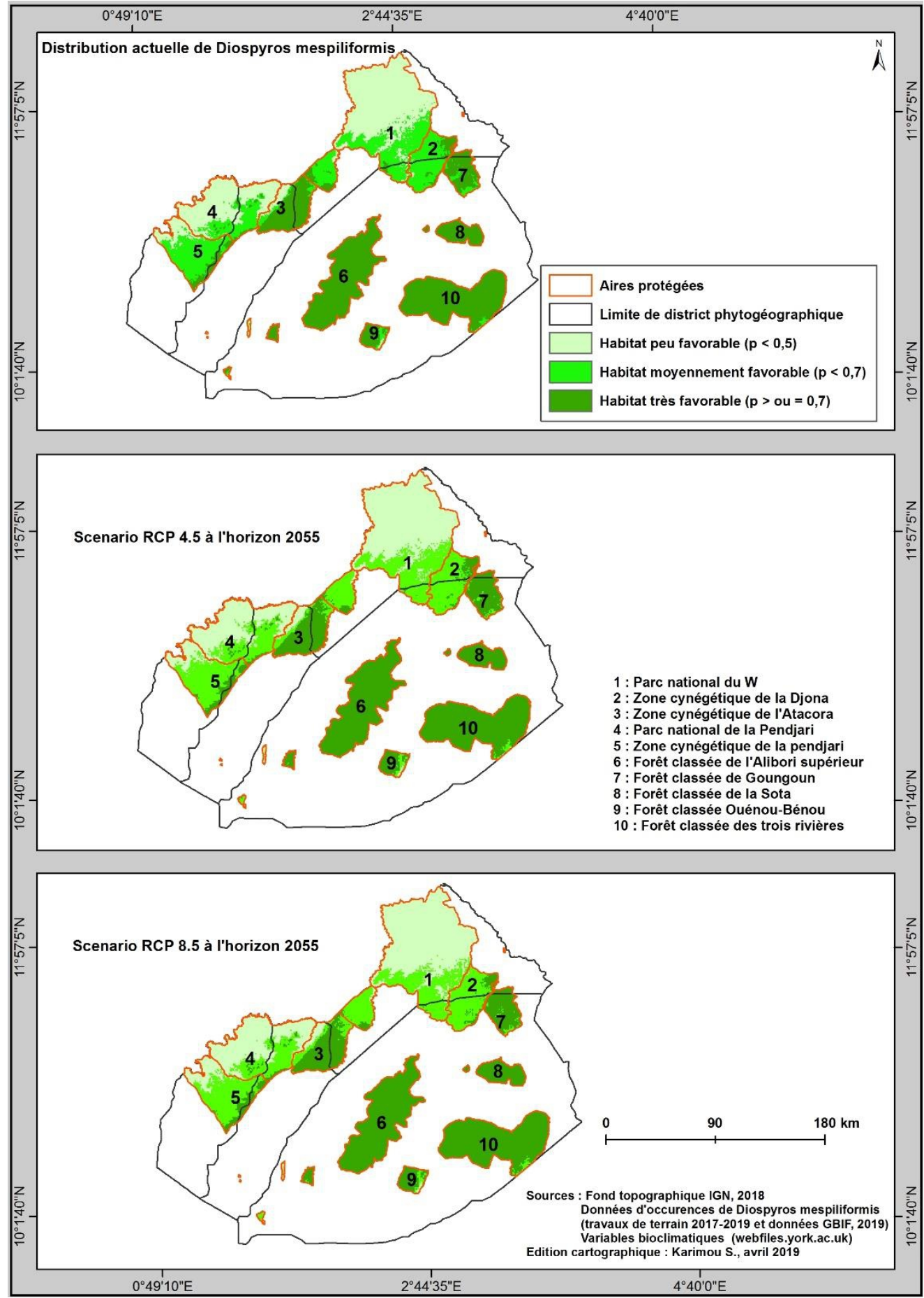

Figure 5 : Spatialisation des habitats de Diospyros mespiliformis dans les aires protégées du milieu d'étude

Source : Résultats de la modélisation 


\section{Discussion}

La modélisation de la niche écologique de Diospyros mespiliformis a été réalisée à partir du programme Maxent (Maximum Entropy) (Phillips et al., 2006). Cette approche est basée sur le concept fondamental de niche de Hutchinson (1957) qui représente les intervalles de conditions et de ressources existant dans un espace donné et qui est potentiellement exploitable par une espèce, sans tenir compte des interactions biotiques possibles avec d'autres espèces (Ricklefs, 2010).

Cinq variables ont été utilisées pour la prédiction de la dynamique spatio-temporelle des habitats de l'espèce dans les conditions climatiques actuelles et futures. Il s'agit des variables telles que le sol (sol), l'évapotranspiration potentielle (pet), la température moyenne annuelle (bio 1), les précipitations du mois le plus sec (bio 14) et l'altitude (alt). Ces variables ont contribuées à la prédiction des habitats de l'espèce en de proportions différentes aussi bien du point de vue de l'ordre de leur intégration dans le modèle que de leur permutation.

L'ordre d'intégration des variables dans le modèle de prédiction des habitats révèle que le paramètre sol introduit dans le modèle a eu un impact positif dans la prédiction des habitats de Disopyros mespiliformis. Selon Badeau et al. (2005), les paramètres édaphiques ont une action physiologique sur les espèces végétales. De plus, le sol constitue un facteur important dans la discrimination de groupements végétaux. Diospyros mespiliformis est une espèce qui s'adapte pratiquement à tout type de sol mais préfère les sols lourds et bien drainés (Arbonier, 2002). La région soudanienne qui est l'objet de la présente étude est caractérisée par une diversité de types de sols qui résultent essentiellement du processus de ferrallitisation et de ferruginisation (Le Barbe et al., 1993 ; Viennot,1978 et Faure, 1977). Ces types de sols en combinaison avec d'autres facteurs du milieu offre donc une diversité de conditions écologiques favorables à la phénologie de Diospyros mespiliformis. Ces résultats sont similaires à ceux obtenus par Adjahossou et al. (2016) et Ayihouenou et al. (2016) sur l'importance de la variable édaphique (sol) dans la prédiction des habitats favorables des espèces végétales.

La réduction de près de $75 \%$ du pouvoir prédictif du modèle observé lorsque les variables telles que l'évapotranspiration (pet) et la température moyenne annuelle (bio1) sont permutées témoigne de leurs rôles plus déterminants dans la prédiction de la dynamique spatio-temporelle des habitats de Diospyros mespiliformis. En effet, ces deux variables agissent de façon directe sur les végétaux et constituent les paramètres climatiques majeurs en écologie des plantes. Ces résultats corroborent ceux de plusieurs auteurs (Dossou et al., 2016 ; Hounkpêvi et al., 2016 et Fandohan et al., 2015) sur le rôle déterminant des paramètres climatiques qui agissent de façon direct 
sur les plantes dans la prédiction de la dynamique spatio-temporelle des habitats des espèces.

Les conditions climatiques actuelles et futurs (RCP 4.5 et 8.5) indiquent que les aires protégées du sud, du centre et de l'est de la région soudanienne sont et demeureront très favorables à la cultures et à la conservation de Diospyros mespiliformis à l'horizon 2055. Par contre, les aires protégées du nord, de l'ouest et des extrêmes nord et ouest sont respectivement moyennement favorable et peu favorable aussi bien dans les conditions climatiques actuelles que futurs. Ce constat peut s'expliquer par le fait que la distribution et l'abondance de Diospyros mespiliformis évoluent suivant le gradient climatique de la région soudanienne. En effet, le gradient climatique de la région soudanienne évolue de façon décroissante du sud vers le nord.

Le potentiel réel actuel des aires de distribution de Diospyros mespiliformis a été sensible aux prédictions des deux scénarii utilisés à 1'horizon 2055. Cela démontre que les changements climatiques peuvent impacter la distribution futur de Diospyros mespiliformis comme l'ont démontré plusieurs auteurs sur la distribution et l'abondance des espèces (Renner et al., 2015 ; Thomas et al., 2015 ; Guisan et Zimmermann, 2000). Par conséquent, l'hypothèse selon laquelle les changements climatiques pourraient modifier l'aire de distribution futur de Diospyros mespiliformis semble être confirmée.

En considérant l'étendue des habitats obtenue par les valeurs de probabilité brut supérieur ou égale à 0,5 généré par le modèle, on peut conclure que quel que soit le scénario utilisé, les changements climatiques ne constitueront qu'une menace mineur à la survie de Disopyros mespiliformis à l'horizon 2055 aussi bien au niveau des aires protégées que dans les terroirs villageois de la région soudanienne. Les caractéristiques climatiques actuelles de son aire de distribution de par le monde prouvent que Diospyros mespiliformis est une espèce qui est capable de survivre sous diverses conditions climatiques contrairement à d'autres espèces. En effet, les précipitations annuelles moyennes dans son aire de répartition varient entre 300 et $2000 \mathrm{~mm}$, avec une saison sèche qui peut s'étendre jusqu'à huit mois. Dans le milieu d'étude, ces valeurs sont comprises en général entre 900 et $1200 \mathrm{~mm}$ avec une saison sèche qui s'étale sur six voire sept mois.

Ainsi, les causes les plus probables de perte considérable d'habitats de cette espèce dans le futur pourraient être dues à la progression du front agricole, la destruction des milieux naturels liée à l'urbanisation et l'exploitation de son bois à divers fins.

\section{Conclusion}

L'évaluation de la niche écologique de Diospyros mespiliformis dans les conditions climatiques actuelles et futures a permis d'examiner la réponse 
de cette espèce face aux variations des conditions climatiques dans le temps. Dans l'ensemble, les conditions écologiques et climatiques de la région soudanienne sont propices à la culture et à la conservation de Diospyros mespiliformis à l'horizon 2055. Il paraît alors impérieux de mener des investigations sur le potentiel de la régénération de cette espèce dans les aires protégées pour l'identification des sites prioritaires à sa culture et conservation dans le futur.

\section{Remerciements}

Les auteurs remercient IDEA WILD pour avoir soutenu la réalisation de cette étude à travers l'octroi d'un Grants à KARIMOU Soufiyanou.

\section{References:}

1. Abdullah, A., Ibrahim, A. A., Sameera, O. B., Ahmad, H. A., Anis, A., Thomas, J. and Mohammad, A. B. (2014). Nucleotide based validation of the endangered plant Diospyros mespiliformis (Ebenaceae) by evaluating short sequence region of plastid $\mathrm{rbcL}$ gene, Plant Omics Journal (POJ) 7(2):102-107 (2014) ISSN:1836-3644.

2. Adjahossou, S. G. C., Gouwakinnou, G. N., Houehanou, D. T., Sode, A. I., Yaoitcha, A. S., Houinato, M. R. B., Sinsin, B. (2016). Efficacité des aires protégées dans la conservation d'habitats favorables prioritaires de ligneux de valeur au Bénin. BOIS ET FORÊTS DES TROPIQUES, N 328 (2), pp. 67 - 76.

3. Adomou, C. A., Sinsin, B., van, der, Maesen, L. J. G. (2006). Phytosociological and chorological approaches to phytogeography: a meso-scale study in Benin. Systematics and Geography of Plants, 76 (2): $155-178$.

4. Agbani, O. P., Gandji, K., Tovissodé, F., Karen, H., Sinsin, B. (2017). Production Fruitière De Quatre Essences Ligneuses Dans La Forêt De Nassou En Zone Soudanienne Du Bénin. European Scientific Journal Vol.13, No.36, $\quad$ pp. $352 \quad-367$. URL:http://dx.doi.org/10.19044/esj.2017.v13n36p352.

5. Ali, A., Issoufou, Hassane, B-A., Dan, G. I., Toudou, D., Abdoul, K., Mahamane, A., Saadou, M. (2017). Effet De Prétraitements, De Substrats Et De Stress Hydriques Sur La Germination Et La Croissance Initiale De Diospyros Mespiliformis Hochst. Ex A.DC. European Scientific Journal, Vol.13, No.21. pp. $251-268$. URL:http://dx.doi.org/10.19044/esj.2017.v13n21p251.

6. Allouche, O, Tsoar, A, Kadmon, R. (2006). Assessing the accuracy of species distribution models: prevalence, kappa and the true skill statistic (TSS). Journal of Applied Ecology 43 (6), pp.1223-1232. http://dx.doi.org/10.1111/j.1365-2664.2006.01214. 
7. Arbonnier, M. (2002). Arbres, arbustes et lianes des zones sèches d'Afrique de l'Ouest. CIRAD (Montpellier) et Muséum National d'Histoire Naturelle (Paris), 574 p.

8. Ayihouenou, E. B., Fandohan, A. B., Sodé, A. I., Gouwakinnou, N. G. et Djossa, A. B. (2016). Biogéographie du néré (Parkia biglobosa (Jack.) R. Br. ex. Don.) sous les conditions environnementales actuelles et futures au Bénin. Bulletin de la Recherche Agronomique du Bénin (BRAB). Pages (pp.) 93-108. ISSN en ligne (on line) : 18407099.

9. Badeau, V., Dupouey, J. L., Clu-Zeau, C., Drapier, J. (2005). Aires potentielles de répartition des essences forestières d'ici 2100. Forêt Entreprise, $\mathrm{n}^{\circ} 162$, pp. 25-29.

10. Belemtougri, R. G., Constantin, B., Cognard, C., Raymond, G., Sawadogo, L. (2006). Effects of two medicinal plants Psidium guajava L. (Myrtaceae) and Diospyros mespiliformis L. (Ebenaceae) leaf extracts on rat skeletal muscle cells in primary culture. J Zhejiang Univ SCIENCE B 7(1):56-63. ISSN 1673-1581.

11. Codjia, J. T. C., Assogbadjo, A. E., Ekué, M. R. M. (2003). Diversité et valorisation au niveau local des ressources végétales forestières alimentaire du Bénin. Cahiers Agricultures 2003, 12 : 1-12.

12. Dossou, E. M., Lougbégnon, T. O., Houessou, L. G. et Codjia, J. T. C. (2016). Analyse de l'impact du changement climatique sur l'aire de distribution actuelle et future de Lannea microcarpa Engl. \& K. Krause au Bénin, Afrique de l'Ouest. Afrique SCIENCE 12(1) (2016) 27 - 38. ISSN 1813-548X

13. Elith, J., C.H. Graham, R.P. Anderson, M. Dudik, S. Ferrier, A. Guisan, R.J. Hijmans, F. Huettmann, J.R. Leathwick, A. Lehmann, J. Li, L.G. Lohmann, B.A. Loiselle, G. Manion, C. Moritz, M. Nakamura, Y. Nakazawa, J.M. Overton, A.T. Peterson, S.J. Phillips, K. Richardson, R. Scachetti-Pereira, R.E. Schapire, J. Soberon, S. Williams, M.S. Wisz, N.E. Zimmermann, (2006). Novel methods improve prediction of species' distributions from occurrence data. Ecography, 29, 129151.

14. Elith, J., Kearney, M. \& Phillips, S. (2010). The art of modelling range-shifting species. Methods in Ecology and Evolution, 1, pp. 330342.

15. Fandohan, AB, Oduor, A. M. O., Sode, AI, Wu, L., Cuni-Sanchez, A, Assede, E, Gouwakinnou, GN. (2015). Modeling vulnerability of protected areas to invasion by Chromolaena odorata under current and future climates. Ecosystem Health and Sustainability 1(6) 20. http://dx.doi.org/10.1890/EHS15-0003.1. 
16. Faure, P. (1977). Notice explicative, carte pédologique de reconnaissance de la République Populaire du Bénin, feuille de Natitingou (6). Office de la Recherche Scientifique et Technique Outre-Mer (ORSTOM) : Paris, $68 \mathrm{p}$.

17. Franklin, J. (2009). Mapping species Distribution: Spatial Inference and Prediction (Ecology, Biodiversity and Conservation) Saint Diego State. Cambridge University Press, $318 \mathrm{p}$.

18. Giam, X., Bradshaw, C. J.A., Hugh, T.W. T., Navjot, S. S. (2010). Future habitat loss and the conservation of plant biodiversity. Biological Conservation 143 (2010) pp.1594-1602.

19. GIEC. (2013). Changement climatiques: les éléments scientifiques. Contribution du Groupe de travail I au $5^{\text {ème }}$ rapport d'évaluation du GIEC. $34 \mathrm{p}$.

20. Gning, O. N., Sarr, O., Gueye, M., Akpo, L.E., Ndiaye, P. M. (2013). Valeur socio-économique de l'arbre en milieu malinké (Khossanto, Sénégal), Journal of Applied Biosciences 70:5617- 5631, ISSN $1997-$ 5902.

21. Guisan, A., Zimmermann, N. E. (2000). Predictive habitat distribution models in ecology. Ecological Modelling, 135: 147-186.

22. Hounkpêvi, A., Tosso, F., Gèmavo, D. S. J. C., Kouassi, E. K., Koné, D. et Glèlè-Kakaï, R. (2016). Climate and potential habitat suitability for cultivation and in situ conservation of the black plum (Vitex doniana Sweet) in Benin, West Africa. International Journal of Agronomy and Agricultural Research (IJAAR). Vol. 8, No. 4, p. 6780. ISSN: 2223-7054 (Print) 2225-3610 (Online).

23. Hutchinson, G.E. (1957). Concluding remarks. Cold Spring Harb. Symp. Quantitative Biology 22: 415-457.

24. Jegede, O., C., Gbadebo, J.O., Adio, A.F., Okesiji, I.T., Akindolu, D.R., Osewa, O.F. (2015). Effect of pretreatment on growth and early seedling performance of diospyros mespiliformis, Journal of Natural Sciences Research, Vol.5, No.2, 2015, ISSN 2224-3186 (Paper) ISSN 2225-0921 (Online).

25. Kumar, S. and Stohlgren, T. J. (2009). Maxent modeling for predicting suitable habitat for threatened and endangered tree Canacomyrica monticola in New Caledonia. Journal of Ecology and Natural Environment Vol. 1(4), pp. 094-098, July, 2009. Available online at http://www.academicjournals.org.

26. Le Barbe, L., Ale, G., Millet, B., Texier, H., Borel, Y. (1993). Monographie des ressources en eaux superficielles de la République du Bénin. Paris, ORSTOM, 540 p.

27. Nchoutpouen, S. (2003). Les produits forestiers non ligneux d'origine végétale dans les provinces du Nord et de l'Extrême Nord du 
Cameroun: inventaire et pratiques locales de gestion. Mémoire ingénieur, FASA, Université de Dschang, 72p.

28. Padalia, H., Srivastava, V., Kushwaha, S.P.S. (2014). Modeling potential invasion range of alien invasive species, Hyptis suaveolens (L.) Poit. in India: Comparison of MaxEnt and GARP. Ecological Informatics 22 (2014) 36-43.

29. Phillips, S. J., Anderson, R. P., Schapire, R. E. (2006). Maximum entropy modeling of species geographic distributions. Ecological Modelling 190 (2006) pp.231-259.

30. Platts, P. J., Marchant, R. A. (2015). AFRICLIM: High-resolution climate projections for ecological applications in Africa. African Journal of Ecology, 53: 103-108.

31. Renner, I.W., Elith, J., Baddeley, A., Fithian, W., Hastie, T., Phillips, S.J., Popovic, G. \& Warton, D.I. (2015). Point process models for presence-only analysis. Methods in Ecology and Evolution, 6, 366379.

32. Ricklefs, R. E. (2010). A Economia da Natureza. Guanabara Koogan, Rio de Janeiro, 570 p.

33. Thomas, B., Lutz, S. \& Rideau, C. (2015). Modélisation de la répartition des campagnols aquatiques du genre Arvicola en Normandie. Groupe Mammalogique Normand, Agence de l'Eau SeineNormandie, 62 p. + annexes.

34. Traoré, L., Ouédraogo, I., Ouédraogo, A., Thiombiano, A. (2011). Perceptions, usages, et vulnérabilité des ressources végétales ligneuses dans le Sud-Ouest du Burkina-Faso. Int. J. Biol. Chem. Sci., 5(1): 258278.

35. Viennot, M. (1978). Notice explicative de la carte pédologique de reconnaissance de la République Populaire Du Bénin A 1/200.000, Feuille de Bembèrèkè. ORSTOM, Paris, France, 45 p.

36. Vivien, J. \& Faure, J. J. (1996). Fruitiers sauvages d'Afrique. Espèces de Cameroun. Editions NGUILA-KEROU, 416 p.

37. Vodouhê, F.G., Coulibaly, O., Greene, C., and Sinsin, B. (2009). Estimating the Local Value of Non-Timber Forest Products to Pendjari Biosphere Reserve Dwellers in Benin. Economic Botany, 63(4), pp. 397412.

38. Yolidjé, I., Alfa, Keita, D., Moussa, I., Toumane, A., Maman, Maarouhi, I., Saley, K., Pirat, J-L., Much, T., Ouamba, J. M. (2019). Caractérisation Phytochimique et Activité Larvicide d'extraits Bruts de Plantes Issues de la Pharmacopée Traditionnelle du Niger sur les Larves d'Anopheles gambiae S.L. European Scientific Journal, Vol.15, No.12, $\quad$ pp. $30 \quad 30 \quad-$ URL:http://dx.doi.org/10.19044/esj.2019.v15n12p30. 
39. Zizka, A., A. Thiombiano, S. Dressler, B. Nacoulma, A.Ouédraogo, I. Ouédraogo, O.Ouédraogo, G. Zizka, K.Hahn \& M. Schmidt (2015). Traditional plant use in BurkinaFaso (West Africa): a national-scale analysis with focus on traditionalmedicine. J. Ethnobiol. Ethnomed. 11: 9 . 